\title{
Efficacy and rapid response of tocilizumab in rheumatoid arthritis patients not responder to previous biologic therapy
}

\begin{abstract}
Objective: To evaluate the rapid response to Tocilizumab (TCZ) treatment in Rheumatoid Arthritis (RA) patients, not responded to a previous biologic therapy.

Methods: We enrolled 29 patients with mild/severe RA (24 women, 5 men) according to the American College Rheumatology 2010 Criteria, who were refractory to Disease Modifying Anti-Rheumatic Drugs (DMARDs), including TNFinhibitors, anti-CD20 and anti-CTL4. They were treated with TCZ $162 \mathrm{mg}$ weekly, Methotrexate (MTX) 10-15mg weekly and low dose prednisone daily $(5-7,5 \mathrm{mg})$. The patients underwent clinical, laboratory, and Power Doppler Ultrasonography (PDUS) evaluation at baseline, 1, 3, 6 months. Efficacy was evaluated based on disease activity and ultrasonographic manifestations of synovitis in the examinated joints.
\end{abstract}

Results: We observed a rapid, statistically significant improvement of both clinical and laboratoristic parameters. After the initial TCZ treatment, the mean DAS 28-ESR (Erythrocyte Sedimentation Rate) decreased from 6,41 at baseline to 2,33 ( $<<0.05)$, and the efficacy has been maintained during this period of TCZ treatment. TCZ treatment decreased the synovitis of affected joints, as measured by ultrasonography. The mean grade of synovitis activity decreased in all patients receiving TCZ treatment $(\Delta-6,80 ; \mathrm{p}<0.05)$. Mean plasma concentrations of total cholesterol, HDL cholesterol and triglycerides raised minimal during the follow up period (Tot cholesterol $\Delta+18.50$, HDL $\Delta+5.80$, triglycerides $\Delta+25.10$ ), but not statistically significant. Rapid and sustained improvements in the acute phase response markers as C Reactiv Protein (CRP) were seen (CRP: $\Delta-4,85 \mathrm{p}<0.05$; haemoglobin $\Delta 1.40 \mathrm{p}<0.05)$. There were greater and statistically significant improvements in physical function, as showed in HAQ-DI $(\Delta-0,155 \mathrm{p}<0.05$ ), SF-36 (ISF $\Delta+6,60 \mathrm{p}<0.05-$ ISM $\Delta+16,10 \mathrm{p}<0.05)$ and FACIT fatigue $(\Delta-11 \mathrm{p}<0.05)$ from baseline, with TCZ treatment.

Conclusion: Our study confirms the rapidity and effectivness of TCZ in treating inflammation in RA patients, both with clinical evaluation and with ultrasonography. PDUS allow a more accurate evaluation of treatment response and a more complete follow-up.

Keywords: Interleukin-6, Rheumatoid arthritis, Synovitis, Ultrasonography, Tocilizumab, DAS28
Volume 8 Issue 3 - 2017

\section{Simone Parisi, Marta Priora, Marco Scarati, Maria Chiara Ditto, Clara Lisa Peroni, Angela Laganà, Enrico Fusaro}

Rheumatology Unit, Azienda Ospedaliero Universitaria Citta della Salute e della Scienza di Torino, Italy

Correspondence: Simone Parisi, Rheumatology Unit, Azienda Ospedaliero Universitaria Citta della Salute e della Scienza di Torino,Turin, Italy Email simone.parisi@hotmail.com

Received: March II, 2017| Published: June 15, 2017
Abbreviations: RA, Rheumatoid Arthritis; IL, Interleukin; DMARDs, Anti-Rheumatic Drugs; DAS, Disease Activity Score; PDUS, Power Doppler ultrasonography; CDAI, Clinical Disease Activity Index; CRP, C-reactive Protein; ESR, Erythrocyte Sedimentation Rate; VAS, Visual Analogic Scale; GC, Glucocorticoids; TCZ, Tocilizumab

\section{Introduction}

Rheumatoid Arthritis (RA) is a systemic chronic inflammatory disease associated with progressive joint damage, pain, fatigue, and disability, affecting approximately $1 \%$ of the population. ${ }^{1}$ Although the exact cause of RA is still unknown, insights into its pathogenesis have confirmed the role of pro-inflammatory cytokines in disease pathways, tumor necrosis factor alpha (TNF $\alpha$ ) interleukin (IL)-1, and IL-6. ${ }^{2,3}$ Interleukin 6 is a pleiotropic cytokine that is over-expressed in synovial tissue in patients with RA, with raised concentrations in serum and synovial fluid. IL- 6 affects the function of neutrophils, T cells, B cells, monocytes, and osteoclast-cells that are highly activated in RA and is the major inducer of the hepatic acute phase response. It is a key feature of RA correlated with disease activity and joint destruction. The effects of IL- 6 are mediated by binding to the IL- 6 receptor (CD126, IL6Rachain), which is expressed on cell surfaces and as a circulating soluble form. ${ }^{4}$

Tocilizumab (TCZ), a recombinant humanised monoclonal IgG1 antihuman IL6 receptor antibody has been shown in previous clinical trials to improve the symptoms of RA and prevent progressive joint destruction among patients with moderate to severe RA refractory to conventional disease modifying anti-rheumatic drugs (DMARDs) when administered either as monotherapy or in combination with conventional DMARDs. Therefore the results of clinical studies suggest that TCZ is a safe and effective alternative for patients who fail to respond to anti-TNF therapy. ${ }^{4-8}$ The disease activity in RA is evaluated by DAS28 (Disease Activity Score), obtained by the examination of 28 tender and swollen joints, Global health analogic scale, and ESR or CRP. ${ }^{9}$ Therefore, evaluating clinical activity in RA patients receiving TCZ is difficult because it blocks IL-6 signalling and rapidly suppresses the serum levels of C-reactive protein (CRP) and erythrocyte sedimentation rate (ESR) which are components of the DAS-28. This could cause an incorrect corresponding between the clinical examination and the inflammatory index, underestimating the disease overview. 
Over the past decade, musculoskeletal ultrasonography has been established as a new imaging modality for assessing RA-affected joints. Ultrasonography has been reported to be more sensitive and reliable than physical examination in the detection of synovial hypertrophy, effusion, and inflammatory activity. ${ }^{10,11}$ Power Doppler ultrasonography (PDUS) in particular detects synovial perfusion in the inflamed joints, and a decrease in composite power Doppler signal score in response to treatment, correlates significantly with DAS28 score and with CRP and ESR, ${ }^{10,12}$ PDUS is also a useful tool in monitoring patients under TNF antagonist therapy and to PDUS findings have a predictive value in radiographic outcomes. ${ }^{13,14}$ TCZ seems to have rapidly positive effects on the pain and joints symptomatology, however this has not been demonstrated in literature yet.

The aim of our study has been in order to evaluate the response to TCZ treatment in RA patients, not responders to previous DMARDs, nor to anti-TNF, anti-CD20, and anti-CTLA4 therapy. We also evaluated laboratory parameters: lipidic pattern (total cholesterol, HDL cholesterol, and triglycerides), haemoglobin, disability, quality of life and ultrasonography analysing synovitis and PD signal.

\section{Material and methods}

This prospective monocentric study was conducted at the Rheumatology Unit of the Azienda Ospedaliero Universitaria of Turin, Italy. All patients signed an informed consent form before the inclusion in the study. The study protocol was approved by the Local Ethical Committee, and data collection and analysis were conducted in accordance with the Declaration of Helsinki.

29 patients with RA (24 women, 5 men) diagnosed according to the American College Rheumatology 2010 Criteria, ${ }^{15}$ who were refractory to DMARDs, including TNF inhibitors, anti-CD20 and anti-CTL4, were enrolled in the study. The mean age of RA patients was 48,7 years $(\mathrm{SD} \pm 8,79)$. The mean disease duration was 5,9 years $(\mathrm{SD} \pm 2,33)$ and the mean of Body Mass Index (BMI) was 23,6 (SD $\pm 2,12$ ). Substantial comorbidity was found in six patients; essential arterial hypertension in four patients, nephrolithiasis in two patients and osteoporosis secondary to long standing glucocorticoids (GC) therapy in two patients. They were treated with TCZ $162 \mathrm{mg}$ weekly, MTX 10-15 mg weekly and low dose prednisone daily (5-7,5mg).

The patients underwent clinical (DAS28, CDAI, VAS Pain, HAQ, SF-36, FACIT fatigue) laboratory (ESR, CRP, Hg, TG, Chol TOT, Chol HDL), and PDUS evaluation at baseline, 1, 3, 6 months. At each visit, patients were evaluated clinically by the same physicians who assessed 28 joints (the bilateral glenohumeral, elbow, and wrist joints, metacarpophalangeal joints, proximal interphalangeal joints of the fingers, and knee joints) for tenderness and swelling. The VAS (100 $\mathrm{mm}$ visual analog scale for patient's general assessment) was rated individually for each patient. Efficacy was evaluated based on disease activity and ultrasonographic manifestations of synovitis in the examinated joints. The disease activity scores and measurements used were the Disease Activity Score 28, using erythrocyte sedimentation rate (DAS28-ESR), Clinical Disease Activity Index (CDAI), C-reactive protein (CRP), erythrocyte sedimentation rate (ESR) and visual analogic scale (VAS). High-frequency ultrasound and power Doppler imaging were performed with an Echographer Esaote $\mathbb{R}$ MyLab70. Of the 28 joints, 24 (excluding bilateral glenohumeral and elbow joints were assessed by PDUS and were scanned by highfrequency ultrasound linear array transducers $(6-18 \mathrm{MHz})$

The images were evaluated for synovial inflammation in the transverse plane and were semi quantitatively assigned a synovitis activity score of grade 0 to grade 3 by a single physician. PDUS was performed by two well-trained rheumatologists: one scanned target joints to obtain images, and both agreed on the assessment of the PD score. The joints were scanned longitudinally and transversally from the dorsal view. PD imaging was performed by selecting a region of interest that included the bony margins and synovial site. ${ }^{16,17} \mathrm{PD}$ signals in each joint were graded on a semi quantitative scale of 0-3 according to OMERACT Criteria (0: absent, no synovial flow: 1: mild, single vessel signal or isolated signals; 2 : moderate, confluent signals in less than half of the synovial area; 3: marked, signals in more than half of the synovial area), corresponding to the maximum score obtained from the synovial sites evaluated in each joint. ${ }^{10}$ Total PD score was calculated as the sum of individual scores for each joint at each examination.

For calculating intra observer reliability, ultrasound investigators scored the PD signals of 50 images randomly selected from stored images and then evaluated again the same selected images arranged in a different order after an interval.

CDAI (Clincal Disease Activity Index): this index uses 28 joint counts, a 28 swollen joint count and a 28 tender joint count but acute phase reactants are not integrated. All of these indices (CDAI, DAS28) include a patient self-report measure. In CDAI is defined as "patient global assessment of disease activity" (PGA) that includes several aspects of health outcomes - that is, also those not or not directly related to disease activity. CDAI include the "evaluator global assessment of disease activity" in addition to the PGA. While the PGA is a subjective measure, the evaluator global integrates subjective and objective measures that are obtained and available to the evaluator. ${ }^{18}$

The Health Assessment Questionnaire-Disability Index (HAQ-DI) was used for assessment of physical function. HAQ-DI assesses the ability to do daily activities by use of 20 questions in eight domains; the final HAQ score is the mean of the highest scores across the eight domains and ranges from 0 to 3 , with higher levels reflecting greater disability. ${ }^{19}$

SF-36 consists of 36 items and eight scales, from which two summary scores can be extracted: the physical component and the mental components scores. The scores range from 0 to 100 (higher scores indicating better health). ${ }^{20}$ The FACIT-Fatigue scale ranges from 0 to 52 and has been validated in the general population, patients with cancer, ${ }^{21}$ and patients with RA; ${ }^{22}$ higher scores represent less fatigue.

Medical Outcomes Study 36-Item Short Form General Health Survey (SF-36), completed at baseline and weeks 24 and Functional Assessment of Chronic Illness Therapy (FACIT)-Fatigue assessment, done at baseline and every 4-12-24 weeks, were used to assess health-related quality of life. The aim of this study is to evaluate both clinically, serologically and with ultrasonography the response to treatment with Tocilizumab in patients with RA refractory to DMARDS and other previous biological treatment.

\section{Statistical analysis}

The data are reported as mean $\pm \mathrm{SD}$. The paired t-test and MannWhitney test were used to test for differences. Correlations between each of the clinical, laboratory, and ultrasound parameters were obtained by Pearson's correlation coefficient. P values less than 0,05 were considered to be statistically significant. Intra observer reliability for the PD score of each joint was estimated by calculating the intra class correlation coefficient (ICC). SPSS ${ }^{\circledR}$ version 17.0 and Microsoft ${ }^{\circledR}$ Office Excel 2007 were used for all analyses. 


\section{Results}

We observed a rapid, statistic significant improvement of both clinical and laboratoristic parameters. All patients were on treatment with low-medium dose of Corticosteroids (Prednisone- equivalent 5-7.5 mg/day) and Methotrexate (MTX) $10-15 \mathrm{mg} /$ week until the end of the study. After the TCZ treatment, the mean DAS28-ESR decreased from 6.41 at baseline to 2.33 (p 0.001 ), and the efficacy has been maintained during this period of TCZ treatment. A more detailed analysis of the changes in individual patients following reinstitution of TCZ treatment is shown in Fig.1 and 2. The mean grade of synovitis of affected joints activity decreased in all patients receiving TCZ treatment $(\Delta-6.80 \mathrm{p}<0.05)$ DAS28 decreased rapidly with TCZ therapy $(\Delta-3,10$ p 0.001$)$

Rapid and sustained improvements in the acute phase response markers as CRP, as well as in haemoglobin, were seen Table 1. Mean CRP concentrations normalised from week 4 of treatment with TCZ in 7 patients $(70 \%)$ and remained below the upper limit of normal until the end of the study Table 2. (Table 1: CRP $\Delta-4.85 \mathrm{p} \mathrm{0.001;} \mathrm{Hg}$ $\Delta 1.40 \mathrm{p} \mathrm{0.046)}$

Mean plasma concentrations of total cholesterol, HDL cholesterol and triglycerides raised minimal during the follow up period (Table 1: Tot Chol $\Delta+18.50$ p 0.165 , HDL Chol $\Delta+5.80$ p $0.645, \mathrm{Tg}$

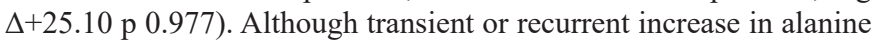
aminotransferase (ALT) and/or aspartate aminotransferase (AST) above the upper limit of normal (ULN; $55 \mathrm{U} / 1)$ were common as patients were also receiving MTX, no patients developed evidence of methotrexate hepatotoxicity. No patient had both a greater than three times ULN increase in ALT and AST and a clinically relevant increase in total bilirubin and there were no cases of hepatitis or clinically significant hepatic dysfunction.

The self patient reported outcome indicated that the strongest effects had already appeared within the first 4 weeks of treatment in pain, morning stiffness and fatigue. There were greater improvements in physical function, as showed in HAQ-DI $(\Delta-0.155 \mathrm{p} 0.051)$, in physical functioning and vitality as well as social functioning and mental health in SF-36 (Physical Score Index PSI $\Delta+6.60-$ Mental Score Index MSI $\Delta+16.10)$ and FACIT fatigue $(\Delta-11 \mathrm{p} 0.034)$ from baseline, with TCZ treatment (Tables 1-3). Fatigue in particular a major debilitating factor in RA - was significantly reduced from baseline after TCZ therapy (Table $1 \&$ Figure 8 ).

Although some patients have been previously treated with other Biologic Drugs at least two years, our data evidenced the rapid positivity response and good efficacy of Tocilizumab after 3 months, confirmed after 6 (Figure 1-3).

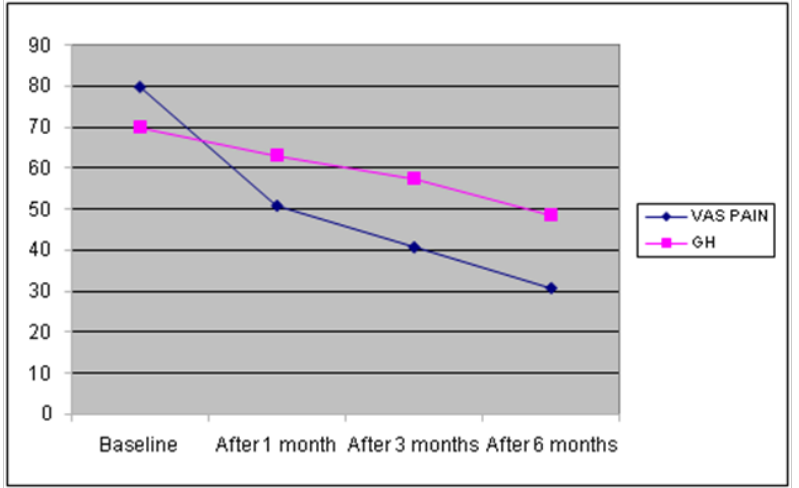

Figure I Baseline and Follow up of Visual Analogic Scale and Global Health.

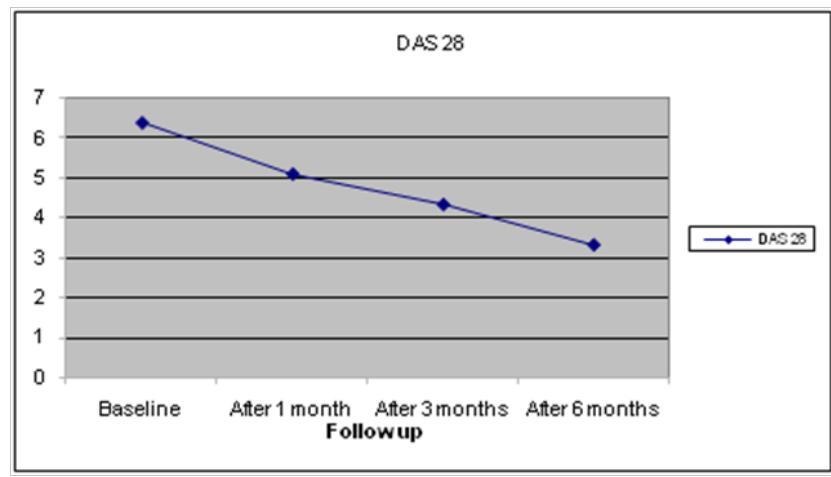

Figure 2 Baseline and Follow up Data of Disease Activity Score (DAS28).

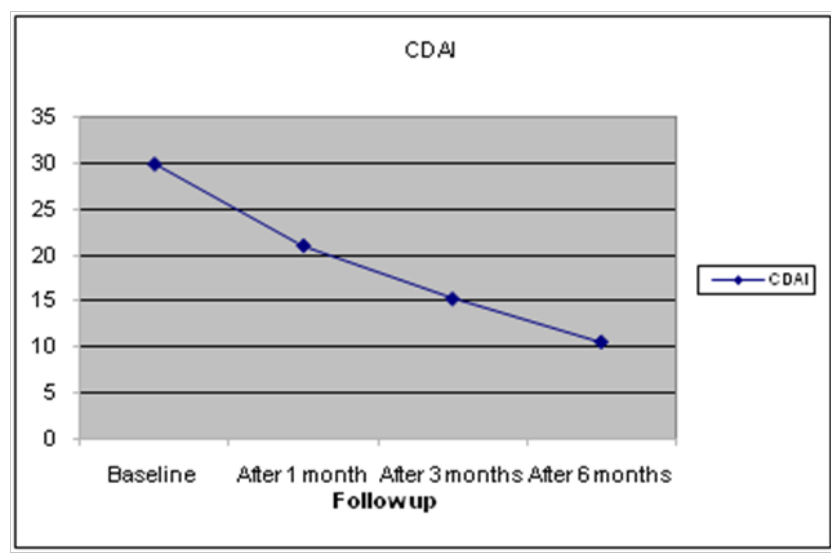

Figure 3 Baseline and Follow up Data of Clinical Activity Index (CDAI).

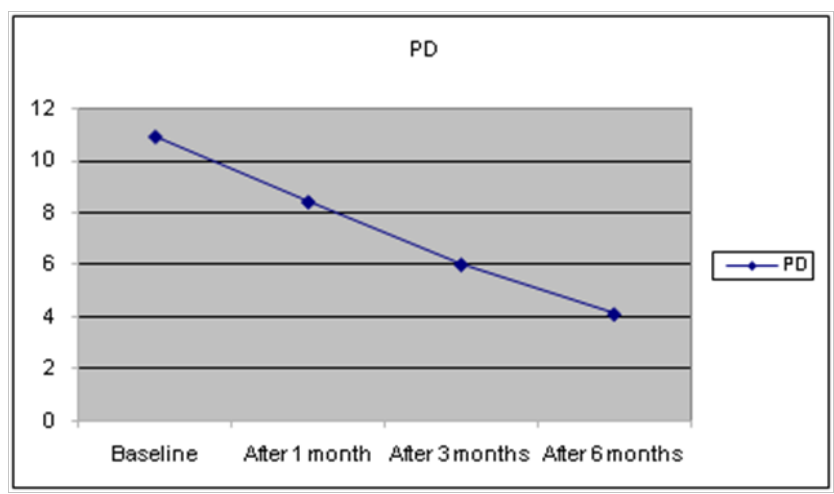

Figure 4 Baseline and Follow up of Power Doppler.

During the study, there were no reported cardiovascular events. No cases of tuberculosis or infections occurred during the study period. All patients completed the study without any severe adverse effects.

\section{Intra observer reliability}

The intra observer ICC for PD signals of each joint was $0.99(95 \%$ confidence interval [95\% CI] 0.98-0.99).

\section{Discussion}

In according to TAMARA Study, ${ }^{5}$ our study suggest that TCZ lead to a rapid improvement of clinical, laboratoristic and instrumental parameters in a patients cohort already treated with other previous DMARDs and other previous biological drugs, underling that this pathology is most likely characterized by many different pathogenic mechanisms. It's important to stress that an early treatment with a 
consequent early improvement in disease activity, lead the patient to a more stable and enduring control, as shown by other studies, ${ }^{8,17,22}$ obtaining a better result also for radiological damage progression. However, the ultrasound images taken before TCZ treatment was reinstituted showed clear signs of moderate to severe synovitis. After treatment with TCZ, most of these signs had disappeared, and those that remained were limited to few spots (Figure 4).

\section{Cardiovascular risk and lipidic pattern}

Low haemoglobin concentrations are indicative of severe chronic inflammation and are reported in $30-40 \%$ of patients with RA, therefore in clinical practice an increase of serum Haemoglobin, as shown in the population of this study, is considered to be a marker of reduction of the systemic inflammatory process.

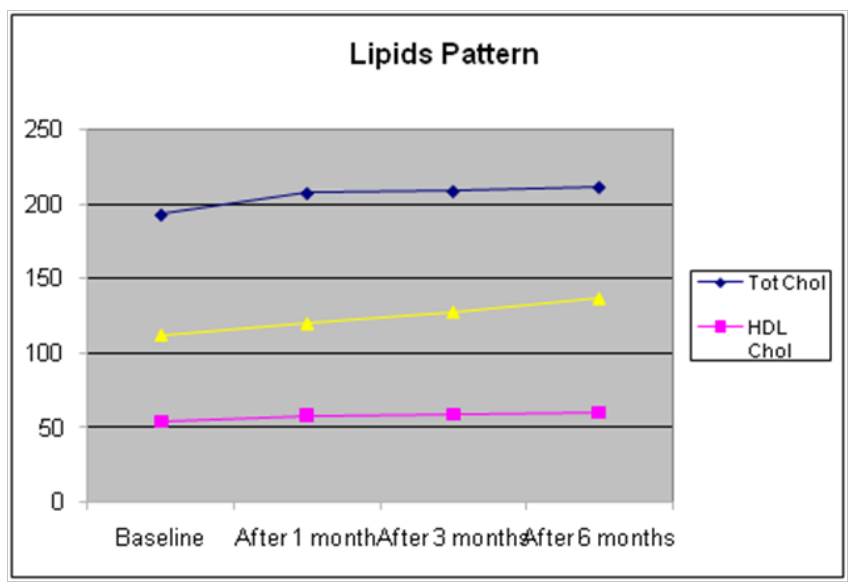

Figure 5 Baseline and Follow up of Lipids Pattern.

Tot Chol: Total Cholesterol; HDL Chol: High Density Lipoprotein Cholesterol; TG: Triglycerides

Since patients with active RA have higher levels of thrombogenic and inflammatory factors, greater oxidative stress, vascular dysfunction and blood pressure, the reduced cardiovascular $(\mathrm{CV})$ risk associated with the use of biological therapy, as TNF inhibitors, is wellknown. ${ }^{24,25}$ Today there are few significant data on CV risk associated with TCZ. Patients with active RA consistently demonstrate reduced HDL-Cholesterol levels and higher Lp(a), and often a higher total cholesterol: HDL cholesterol ratio, ${ }^{26}$ suggesting a greater and more consistent reduction in HDL-cholesterol. However, some studies also report lower total and LDL-cholesterol levels in RA. ${ }^{27-29}$

Reduced HDL-cholesterol and elevated Lp(a) correlate with elevated serum CRP levels and thus inflammatory activity in RA. ${ }^{30}$ Interestingly, inflammatory activation may also drive lower total and LDL-cholesterol levels in RA. ${ }^{29-30}$ Similarly to other chronic inflammatory clinical conditions, also in RA, HDL cholesterol and total cholesterol levels are often reduced; these changes are frequently in line with inflammatory marker elevations. In humans several inflammatory reactive cytokines are known to reduce both HDL cholesterol and LDL cholesterol levels; activation of the reticulo endothelial system is likely associated with such changes, but other mechanisms may be involved. . $^{29,31,32}$

Consequently, inflammation reduction after pharmacological treatments (DMARDs, biologic therapy) may increase lipid levels (not only HDL, but also total and LDL cholesterol). Therefore, changes in lipid profiles, particularly the elevation in cholesterol and triglycerides levels that occur with treatments for severe inflammation, may not represent increased cardiovascular risk as in the usual understanding of lipid-level elevations in individuals without significant inflammation. ${ }^{33-35}$ Rather, such changes in lipid levels, in part or largely, may represent a predictable response to attenuation of inflammation. Finally, in clinical practice, the current advice on the use of lipid profiles in any patient with RA is ${ }^{33}$ to measure both total cholesterol and HDL cholesterol and to use the total cholesterol: HDL cholesterol ratio in conjunction with other established risk factors for the calculation of absolute $\mathrm{CV}$ risk, and ${ }^{27}$ to treat accordingly when the absolute $\mathrm{CV}$ risk is high. ${ }^{29}$

Interestingly, increases from lower than normal base line lipid concentrations have been observed with other efficacious therapies including TNF inhibitors. ${ }^{34,35}$ Some studies, based on reduction of inflammatory response in RA, have shown decreased rates of cardiovascular events, despite the increase in lipid concentration. ${ }^{36,37}$

Increase in total and low-density lipoprotein cholesterol levels in this study were low previous reports ${ }^{22,38,39}$ and were associated with marked decreases in CRP. ${ }^{40,41}$ Considering the recognised higher incidence of cardiovascular mortality in RA patients, ${ }^{30,34,42}$ traditional risk factors for cardiovascular disease, including dyslipidemia, are not necessarily predictive of events for patients with RA. ${ }^{43}$

\section{Disability and quality of life}

Few studies reported the impact of biotherapies on fatigue. The effect of biotherapies on fatigue in RA is poor. ${ }^{44}$ Data from recent trials of biological agents demonstrate that these agents can reverse diseaserelated decrements in productivity and limitations in participation in family, social and leisure activities in patients with active RA. Furthermore, as reflected by the HAQ (Table 1 \& Figure 6) and SF-36 (Table 2,3 \& Figure 7), the quality of life also improved (Figure 6 \& 7). Our study has some limitations: patient's heterogeneity like the duration of disease, differences in previously therapy and the lack of a control group.

Table I Baseline and Follow up data

\begin{tabular}{llllll}
\hline Parameters & Baseline & $\begin{array}{l}\text { After I } \\
\text { Month }\end{array}$ & $\begin{array}{l}\text { After } 3 \\
\text { Months }\end{array}$ & \multicolumn{2}{c}{ After 6 Months P Value } \\
\hline DAS 28 & $6,41 \pm 0,79$ & $5,12 \pm 0,69$ & $4,35 \pm 0,72$ & $3,33 \pm 0,58$ & 0,001 \\
CDAI & $29,90 \pm 6,45$ & $21,00 \pm 6,45$ & $15,2 \pm 5,92$ & $10,5 \pm 4,30$ & 0,032 \\
VAS Dolore & $8,00 \pm 0,41$ & $5,95 \pm 0,55$ & $4,80 \pm 0,59$ & $3,85 \pm 0,67$ & 0,002 \\
GH & $70 \pm 6,24$ & $63,00 \pm 4,22$ & $57,50 \pm 3,53$ & $48,5 \pm 4,12$ & 0,044 \\
CRP & $6,78 \pm 2,83$ & $3,23 \pm 1,14$ & $1,82 \pm 0,76$ & $1,93 \pm 0,64$ & 0,001 \\
HAQ & $0,94 \pm 0,15$ & $0,89 \pm 0,12$ & $0,82 \pm 0,13$ & $0,78 \pm 0,11$ & 0,051 \\
FACIT & $26,8 \pm 2,66$ & $22,5 \pm 2,12$ & $18,9 \pm 2,23$ & $15,8 \pm 3,33$ & 0,034 \\
PD & $10,9 \pm 1,37$ & $8,4 \pm 1,26$ & $6 \pm 1,15$ & $4,1 \pm 0,87$ & 0,011 \\
Hg & $13,36 \pm 0,83$ & $13,93 \pm 0,50$ & $14,3 \pm 0,45$ & $14,76 \pm 0,48$ & 0,046 \\
Tot Chol & $192,9 \pm 14,15$ & $207,3 \pm 15,83$ & $208,7 \pm 14,82$ & $211,4 \pm 12,83$ & 0,165 \\
HDL Chol & $54,1 \pm 9,82$ & $58,1 \pm 9,12$ & $59,1 \pm 9,30$ & $59,9 \pm 7,44$ & 0,645 \\
TG & $111 \pm 42,15$ & $207,3 \pm 38,45$ & $127,1 \pm 32,93$ & $136,5 \pm 30,14$ & 0,977 \\
\hline
\end{tabular}

DAS28: Disease Activity Score; CDAI: Clinical Disease Activity Index; VAS: Visual Analogic Scale Pain; GH: Global Health; HAQ: Health Assessment Questionnaire; FACIT;PD:Power Doppler; Hg: Haemoglobin; otal Cholesterol; HDL Cholesterol;TG triglycerides

Table 2 Baseline and Follow up Data of Short Form General Health Survey

\begin{tabular}{lllllll}
\hline \multicolumn{7}{l}{ SF-36 Overall Data } \\
\hline PSI 0 & MSI 0 & PSI 6 & MSI 6 & $\Delta$ PSI & $\Delta$ MSI \\
Mean & 33,10 & 33,50 & 39,70 & 46,60 & 6,60 & 13,10 \\
St Dev & 3,93 & 3,95 & 3,65 & 3,95 & 3,78 & 4,79 \\
\hline
\end{tabular}

PSI: Physical Score Index; MSI: Mental Score Index 
Table 3 Baseline and Follow up Data of Short Form General Health Survey

\begin{tabular}{|c|c|c|c|c|c|c|}
\hline \multicolumn{7}{|c|}{ SF-36 Bias From The Italian Population } \\
\hline & PSI DS 0 & MSI DS 0 & PSI DS 6 & MSI DS 6 & $\triangle$ PSI DS & $\Delta \mathrm{MSI} D \mathrm{~S}$ \\
\hline Mean & $-1,69$ & $-1,65$ & $-1,03$ & $-0,34$ & 0,66 & $|, 3|$ \\
\hline St Dev & 0,39 & 0,40 & 0,37 & 0,39 & 0,38 & 0,48 \\
\hline
\end{tabular}

PSI: Physical Score Index; MSI: Mental Score Index

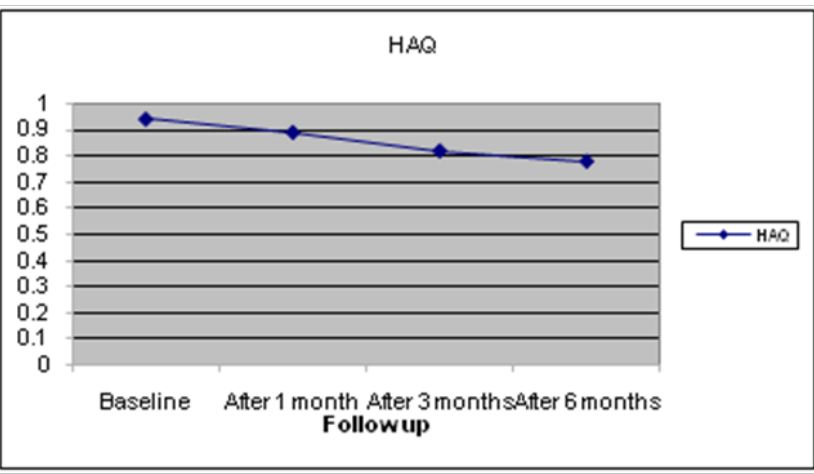

Figure 6 Baseline and Follow up of Health Assessment Questionnaire (HAQ).

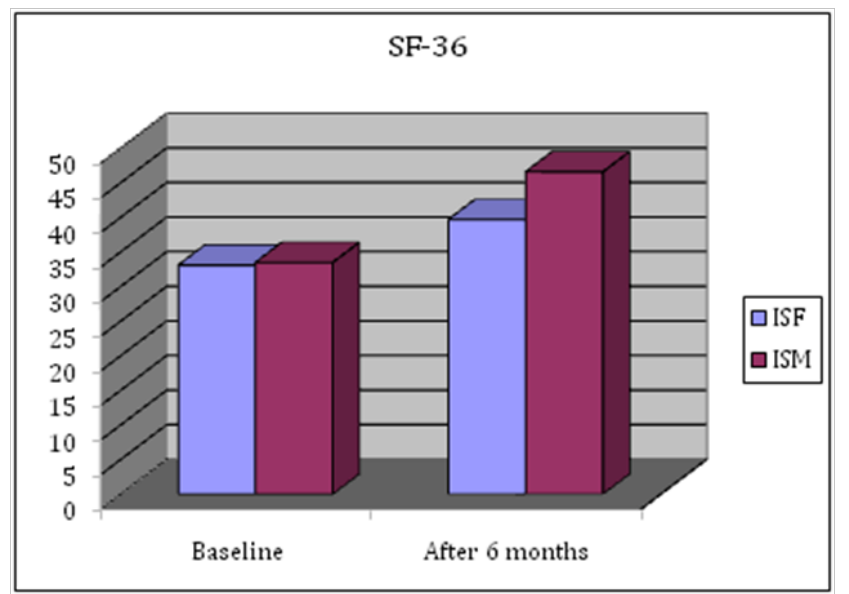

Figure 7 Baseline and Follow up of Short Form General Health Survey (SF36).

PSI: Physical Synthetic Index; MSI: Mental Synthetic Index

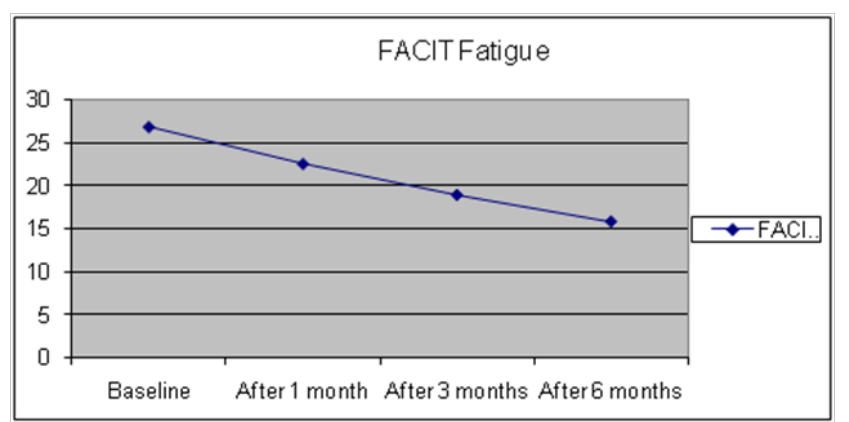

Figure 8 Baseline and follow up of FACIT-Fatigue.

\section{Conclusion}

Our study confirms the rapid response and the effectivness of TCZ to treating inflammation in RA patients, both with clinical, serological and ultrasound evaluation. Therefore, PDUS allow a more accurate evaluation of treatment response and a more complete follow-up, especially because is more precise and objective than clinimetric index in joint damage prediction.

\section{Acknowledgments}

None.

\section{Conflicts of interest}

None.

\section{References}

1. Gabriel SE. The epidemiology of rheumatoid arthritis. Rheum Dis Clin North Am. 2001;27(2):269-281.

2. Smolen JS, Aletaha D, Koeller M, et al. New therapies for treatment of rheumatoid arthritis. Lancet . 2007;370(9602):1861-1874.

3. Firestein GS. Evolving concepts of rheumatoid arthritis. Nature 2003;423(6937):356-361.

4. Smolen JS, Beaulieu A, Rubbert-Roth A, et al. OPTION Investigators. Effect of interleukin-6 receptor inhibition with tocilizumab in patients with rheumatoid arthritis (OPTION study): a double-blind, placebocontrolled, randomised trial. Lancet. 2008;371(9617):987-997.

5. Burmester GR, Feist E, Kellner H, et al. Effectiveness and safety of the interleukin 6-receptor antagonist tocilizumab after 4 and 24 weeks in patients with active rheumatoid arthritis: the first phase IIIb real-life study (TAMARA). Ann Rheum Dis. 2011;70(5):755-759.

6. Emery P, Keystone E, tony HP, et al. IL -6 receptor inhibition with tocilizumab improves treatment outcomes in patients with rheumatoid arthritis refractory to anti-tumor necrosis factor biologicals: results from a 24-week multicentre randomised placebo-controlled trial. Ann Rheum Dis. 2008;67(11):1516-1523.

7. Fleischmann RM, Burgos-Vargas R, Ambs P LITHE. Tocilizumab inhibits radiographic progression and improves physical function in Rheumatoid arthritis (RA) patients (Pts) at 2 Yrs with increasing clinical efficacy over time. Abstract 637. Oral presentation at Amercan College of Rheumatology. 2009.

8. Nishimoto N, Hashimoto J, Miyasaka N, et al. Study of active controlled monotherapy used for rheumatoid arthritis, an IL-6 inhibitor (SAMURAI): evidence of clinical and radiographic benefit from an $\mathrm{x}$ ray reader-blinded randomised controlled trial of tocilizumab. Ann Rheum Dis. 2007;66(9):1162-1167.

9. Prevoo ML, van't Hof MA, Kuper HH, et al. Modified disease activity scores that include ywenty-eight-joints counts. Development and validation in a prospective longitudinal study of patients with rheumatioioid arthritis. Arthritis Rheum. 1995;38(1):44-48.

10. Naredo E, Bonilla G, Gamero F, et al. Assessment of inflammatory activity in rheumatoid arthritis: a comparative study of clinical evaluation with grey scale and power Doppler ultrasonography. Ann Rheum Dis. 2005;64(3):375-381.

11. Kane D, Balint PV, Sturrock RD. Ultrasonography is superior to clinical examination in the detection and localization of knee joint effusion in rheumatoid arthritis. J Rheumatol. 2003;30(5):966-971.

12. Hameed B, Pilcher J, Heron C, et al. The relation between composite ultrasound measures and the DAS28 score, its components and acute phase markers in adult RA. Rheumatology (Oxford). 2008;47(4):476-480.

13. Iagnocco A, Perella C, Naredo E, et al. Etanercept in the treatment of rheumatoid arthritis: clinical follow-up over one year by ultrasonography. Clin Rheumatol. 2008;27(4):491-496.

14. Terslev L, Torp-Pedersen S, Qvistgaard E, et al. Effects of treatment with etanercept (Enbrel, TNRF:Fc) on rheumatoid arthritis evaluated by Doppler ultrasonography. Ann Rheum Dis. 2003;62(2):178-181.

15. Aletaha D, Neogi T, SIlman A, et al. 2010 rheumatoid arthritis classification criteria: an America College of Rhuematology/European League Against Rheumatism collaborative initiative. Ann Rheum Dis. 2010;69(9):1580-1588. 
16. Wakefield RJ, Balint PV, Szkudlarek M, et al. Musculoskeletal ultrasound including definitions for ultrasonographicpathology. $J$ Rheumatol. 2005;32(12):2485-2487.

17. Backhaus M, Burmester GR, Gerber T, et al. Guidelines for musculoskeletal ultrasound in rheumatology. Ann Rheum Dis. 2001;60(7):641-649.

18. Aletaha D, Smolen J. The Simplified Disease Activity Index (SDAI) and the Clinical Disease Activity Index (CDAI): a review of their usefulness and validity in rheumatoid arthritis. Clin Exp Rheumatol. 2005;23(5 Suppl 39):S100-S108.

19. Bruce B, Fries JF. The Stanford Helath Assessment Questionnaire: a review of its history, issues, progress, and documentation. J Rheumatol. 2003;30(1):167-178.

20. Ware JE Jr, Sherbourne CD. The MOS 36-item short-form health survey (SF-36). I. Conceptual framework and item selection. Med Care. 1992;30(6):473-483.

21. Cella D, Lai JS, Chang CH, et al. Fatigue in cancer patients compared with fatigue in the General United States population. Cancer. 2002;94(2):528-538.

22. Cella D, Yount S, Sorensen M, et al. Validation of the Functional Assessment of Chronic Illness Therapy Fatigue Scale relative to other instrumentation in patients with rheumatoid arthritis. J Rheumatol. 2005;32(5):811-819.

23. Al-Aly Z, Pan H, Zeringue A, et al. Tumor necrosis factor- $\alpha$ blockade, cardiovascular outcome, and survival in rheumatoid arthritis. Transl Res. 2011;157(1):10-18

24. Sattar N, McCarey DW, Capell H, et al. Explaining how "high-grade" systemic inflammation accelerates vascular risk in rheumatoid arthritis. Circulation. 2003;108(24):2957-2963.

25. Stampfer MJ, Sacks FM, Salvini S, et al. A prospective study of cholesterol, apolipoproteins, and the risk of myocardial infarction. $N$ Engl J Med. 1991;325(6):373-381.

26. Situnayake RD, Kitas G. Dyslipidemia and rheumatoid arthritis. Ann Rheum Dis. 1997;56:341-342.

27. Park YB, Lee SK, Suh CH, et al. Lipid profiles in untreated patients with rheumatoid arthritis. J Rheumatol. 1999;26(8):1701-1704.

28. Choy E, Sattar N. Interpreting lipid levels in the context of highgrade inflammatory states with a focus on rheumatoid arthritis: a challenge to conventional cardiovascular risk actions. Ann Rheum Dis. 2009;68(4):460-469.

29. Dursunoglu D, Evrenglul H, Polat B, et al. Lp(a)lipoprotein and lipids in patients with rheumatoid arthritis:serum levels and relationship to inflammation. Rheumatol Int. 2005;25(4):241-245.

30. Vermont CL, den Brinker M, Kakeci N, et al. Serum lipids and disease severity in children with severe meningococcal sepsis. Crit Care Med. 2005;33(7):1610-1615.
31. Alexopoulos CG, Poumaras S, Vaslamatzis M, et al. Changes in serum lipids and lipoproteins in cancer patients during chemotherapy. Cancer Chemother Pharmacol. 1992;30(5):412-416.

32. Weinblatt ME, Kuritzky L. RAPID: rheumatoid arthritis. J Fam Pract. 2007;56(4 Supp1):S1-S7.

33. Sattar N, Crompton P, Cherry L, et al. Effects of tumor necrosis factor blockade on cardiovascular risk factors in psoriatic arthritis: a doubleblind, placebo-controlled study. Athritis Rheum. 2007;56(3):831-839.

34. Allanore Y, Kahan A, Sellam J, et al. Effects of repeated infliximab therapy on serum lipid profile in patients with refractory rheumatoid arthritis. Clin Chim Acta. 2006;365(1-2):143-148.

35. Choi HK, Hernan MA, Seeger JD, et al. Methotrexate and mortality in patients with rheumatoid arthritis:a prospective study. Lancet 2002;359(9313):1173-1177.

36. Wolfe F, Michaud K. Heart failure in rheumatoid arthritis:rates, predictors, and the effect of anti-tumor necrosis factor therapy. Am J Med. 2004;116(5):305-311.

37. Maini RN, Taylor PC, Szechinski J, et al. Double-blind randomized controlled clinical trial of the interleukin-6 receptor antagonist, tocilizumab, in European patients with rheumatoid arthritis who had an incomplete response to methotrexate. Arthritis Rheum. 2006;54(9):2817-2829.

38. Smolen JS, Beaulieu A, Rupert-Roth A, et al. Effect of interleukin-6 receptor inhibition with tocilizumab in patients with rheumatoid arthritis (OPTION study): a double-blind, placebo-controlled, randomised trial. Lancet. 2008;371(9617):987-997.

39. Nishimoto N, Yoshizaki K, Miyasaka, et al. Treatment of rheumatoid arthritis with humanized anti-interleukin-6 receptor antibody: a multicenter, double-blind, placebo-controlled trial. Arthritis Rheum. 2004;50(6):1761-1769.

40. Dessein PH, Stanwix AE, Joffe BI. Cardiovascular risk in rheumatoid arthritis versus osteoarthritis : acute phase response related decreased insulin sensitivity and high-density lipoprotein cholesterol as well as clustering of metabolic syndrome features in rheumatoid arthritis. Arthritis Res. 2002;4(5):R5.

41. Situnayake RD, Kitas G. Dyslipidemia,Rheumatoid Arthritis. Ann Rheum Dis. 1997;56:341-342.

42. Chen YF, Jobaputra P, Barton P, et al. A systematic review of the effectiveness of adalimumab, etanercept and infliximab for the treatment of rheumatoid arthritis in adults and an economic evaluation of their cost-effectiveness. Health Technol Assess. 2006;10:1-245.

43. Lennart T H Jacobsson, Carl Turesson, Jan-Åke Nilsson, et al. Treatmen with TNF blockers and mortality risk in patients with rheumatoid arthritis. Ann Rheum Dis. 2007;66(5):670-675.

44. Chauffier K, Salliot C, Berenbaum F, et al. Effect of biotherapies on fatigue in rheumatoid arthritis: a systematic review of the literature and meta-analysis. Rheumatology (Oxford). 2011. 